\title{
The three-quark potential and perfect Abelian dominance in SU(3) lattice QCD
}

\section{Hideo Suganuma*,}

Department of Physics \& Division of Physics and Astronomy, Graduate School of Science, Kyoto University, Kitashirakawaoiwake, Sakyo, Kyoto 606-8502, Japan

E-mail: suganuma@scphys.kyoto-u.ac.jp

\section{Naoyuki Sakumichi}

Ochanomizu University, 2-1-1 Otsuka, Bunkyo, Tokyo 112-8610, Japan

We study the static three-quark (3Q) potential for more than 300 different patterns of 3Q systems with high statistics, i.e., 1000-2000 gauge configurations, in SU(3) lattice QCD at the quenched level. For all the distances, the $3 \mathrm{Q}$ potential is found to be well described by the Y-ansatz, i.e., one-gluon-exchange (OGE) Coulomb plus Y-type linear potential. Also, we investigate Abelian projection of quark confinement in the context of the dual superconductor picture proposed by Yoichiro Nambu et al. in SU(3) lattice QCD. Remarkably, quark confinement forces in both QQ and 3Q systems can be described only with Abelian variables in the maximally Abelian gauge, i.e., $\sigma_{\mathrm{QQ}} \simeq \sigma_{\mathrm{QQ}}^{\mathrm{Abel}} \simeq \sigma_{3 \mathrm{Q}} \simeq \sigma_{3 \mathrm{Q}}^{\mathrm{Abel}}$, which we call "perfect Abelian dominance" of quark confinement.

The 33rd International Symposium on Lattice Field Theory

14 -18 July 2015

Kobe International Conference Center, Kobe, Japan

${ }^{*}$ Speaker. 


\section{Introduction}

In 1966, Yoichiro Nambu [1] first proposed the SU(3) gauge theory, i.e., quantum chromodynamics (QCD), as a field theory of quarks, just after the introduction of the color quantum number [2]. In 1973, the asymptotic freedom of QCD was theoretically shown [3], and QCD was established as the fundamental theory of the strong interaction. While perturbative QCD works well at high energies, infrared QCD exhibits strong-coupling nature and various nonperturbative phenomena such as dynamical chiral-symmetry breaking [4] and color confinement [5].

Among the nonperturbative properties of QCD, color confinement is one of the most difficult important subjects. The difficulty is considered to originate from non-Abelian dynamics and nonperturbative features of QCD, which are largely different from QED. However, it is not clear whether quark confinement is peculiar to the non-Abelian nature of QCD or not.

On the quark confinement in hadrons, QQ systems have been well investigated in lattice QCD [6], but the quark interaction in baryonic three-quark (3Q) systems [7 8] has not been studied so much. Note however that the nucleon is one of the main ingredients of the matter in our real world, and therefore the quark confinement in baryons would be fairly important. Furthermore, the threebody force among three quarks is a "primary" force reflecting the SU(3) gauge symmetry in QCD [7], while the three-body force appears as a residual interaction in most fields of physics.

In this paper, we accurately measure the static $3 \mathrm{Q}$ potential and quark confinement in baryons in SU(3) quenched lattice QCD with 1000-2000 gauge configurations [9]. In parallel, we also investigate Abelian projection of quark confinement for both QQ and 3Q systems [9, 10].

\section{Dual Superconductor Picture and Maximally Abelian projection}

In 1970's, Nambu, 't Hooft and Mandelstam proposed a dual-superconductor theory for quark confinement [5]. In this theory, the QCD vacuum is identified as a color-magnetic monopole condensed system, and there occurs one-dimensional squeezing of the color-electric flux among (anti)quarks by the dual Meissner effect, which leads to the string picture [11] of hadrons.

However, there are two large gaps between QCD and the dual-superconductor picture [12].

1. The dual-superconductor picture is based on the Abelian gauge theory subject to the Maxwelltype equations, while QCD is a non-Abelian gauge theory.

2. The dual-superconductor picture requires color-magnetic monopole condensation as the key concept, while QCD does not have such a monopole as the elementary degrees of freedom.

As a connection between the dual superconductor and QCD, 't Hooft proposed "Abelian projection" [13, 14, which accompanies topological appearance of magnetic monopoles. 't Hooft also conjectured that long-distance physics such as confinement is realized only by Abelian degrees of freedom in QCD [13], which is called "(infrared) Abelian dominance". Actually, in the maximally Abelian (MA) gauge [15], infrared QCD becomes Abelian-like [16] as a result of a large off-diagonal gluon mass of about $1 \mathrm{GeV}$ [17], and also there appears a large clustering of the monopole-current network in the QCD vacuum [15, 18]. In fact, by taking the MA gauge, infrared QCD seems to resemble an Abelian dual-superconductor system. In the MA gauge, Abelian dominance of quark confinement has been investigated mainly for QQ̄ systems in SU(2) and SU(3) lattice QCD [16, 19, 20]. 
Lattice QCD is described with the link variable $U_{\mu}(s)=e^{i a g A_{\mu}(s)}$ (a: lattice spacing, $g$ : gauge coupling, $A_{\mu}$ : gluon fields), and SU(3) MA gauge fixing [9 10] is performed by maximizing

$$
R_{\mathrm{MA}}\left[U_{\mu}(s)\right] \equiv \sum_{s} \sum_{\mu=1}^{4} \operatorname{tr}\left(U_{\mu}^{\dagger}(s) \vec{H} U_{\mu}(s) \vec{H}\right)=\frac{1}{2} \sum_{s} \sum_{\mu=1}^{4}\left(\sum_{i=1}^{3}\left|U_{\mu}(s)_{i i}\right|^{2}-1\right),
$$

under the $\mathrm{SU}(3)$ gauge transformation. In our calculation, we numerically maximize $R_{\mathrm{MA}}$ using the over-relaxation method $9,10,19$. The converged value of $\left\langle R_{\mathrm{MA}}\right\rangle /(4 \mathrm{~V}) \in\left[-\frac{1}{2}, 1\right]$ (V: lattice volume) is, e.g., $0.7072(6)$ at $\beta=5.8$ and $0.7322(5)$ at $\beta=6.0$, and the maximized value of $R_{\mathrm{MA}}$ is almost the same over 1000-2000 gauge configurations. Then, our procedure seems to escape bad local minima, where $R_{\mathrm{MA}}$ is relatively small, so that the Gribov copy effect would not be significant.

The Abelian link variable $u_{\mu}(s)=e^{i \theta_{\mu}^{3}(s) T_{3}+i \theta_{\mu}^{8}(s) T_{8}} \in \mathrm{U}(1)^{2}$ is extracted from the link variable $U_{\mu}^{\mathrm{MA}}(s) \in \mathrm{SU}(3)$ in the MA gauge, by maximizing $R_{\text {Abel }} \equiv \frac{1}{3} \operatorname{Re} \operatorname{tr}\left(U_{\mu}^{\mathrm{MA}}(s) u_{\mu}^{\dagger}(s)\right) \in\left[-\frac{1}{2}, 1\right]$ 国.

\section{The quark-antiquark potential and perfect Abelian dominance of confinement}

First, we investigate the QQ potential $V(r)$ in SU(3) quenched lattice QCD on $L^{3} \times L_{t}$, with $\left(\beta, L^{3} L_{t}\right)=\left(6.4,32^{4}\right),\left(6.0,32^{4}\right)$ and $\left(5.8,16^{3} 32\right)$ [10]. The static QQ potential $V(r)$ is obtained from the Wilson loop [6], and its MA projection (Abelian part) $V_{\text {Abel }}(r)$ is similarly defined as

$$
V(r)=-\lim _{t \rightarrow \infty} \frac{1}{t} \ln \left\langle W\left[U_{\mu}\right]\right\rangle_{r \times t}, \quad V_{\text {Abel }}(r)=-\lim _{t \rightarrow \infty} \frac{1}{t} \ln \left\langle W\left[u_{\mu}\right]\right\rangle_{r \times t} .
$$

(We also define the off-diagonal part $V_{\text {off }}(r)$, and numerically find $V(r) \simeq V_{\text {Abel }}(r)+V_{\text {off }}(r)[10]$.)

We show in Fig. 1 the lattice QCD result of the QQ potential $V(r)$ and its Abelian part $V_{\text {Abel }}(r)$. They are found to be well reproduced by the Coulomb-plus-linear ansatz, respectively:

$$
V(r)=-\frac{A}{r}+\sigma r+C, \quad V_{\text {Abel }}(r)=-\frac{A_{\text {Abel }}}{r}+\sigma_{\text {Abel }} r+C_{\text {Abel }} .
$$

Remarkably, we find "perfect Abelian dominance" of the string tension, $\sigma_{\text {Abel }} \simeq \sigma$, on these lattices.

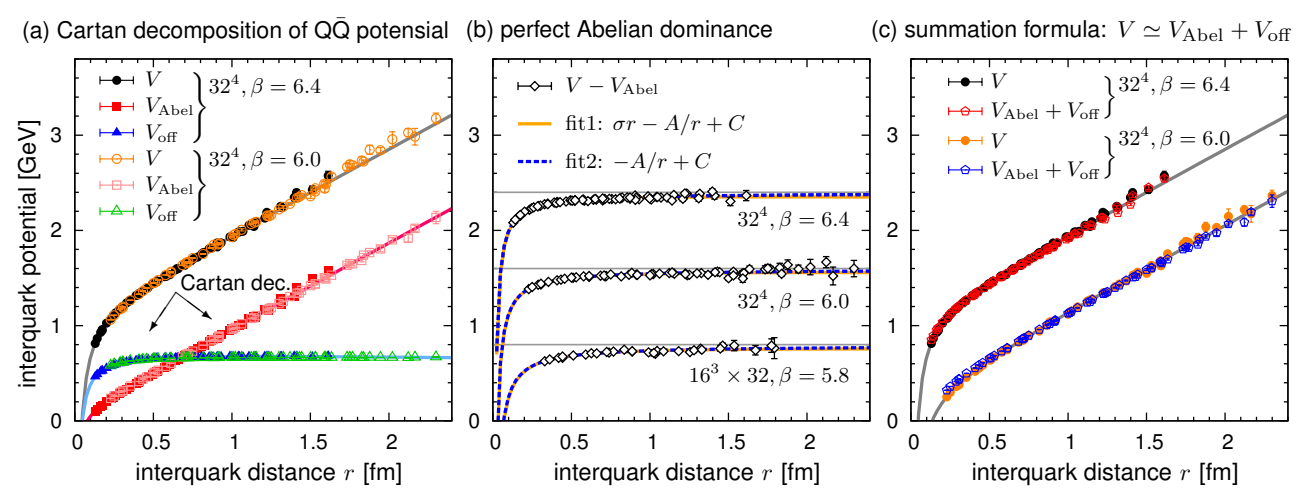

Figure 1: (a) Cartan decomposition of the static QQ potential $V(r)$ (circles) into the Abelian part $V_{\text {Abel }}(r)$ (squares) and the off-diagonal part $V_{\text {off }}(r)$ (triangles) on $32^{4}$ at $\beta=6.4$ (filled) and 6.0 (open). For each potential, the best-fit Coulomb-plus-linear curve is added. (b) Fit analysis of $V(r)-V_{\text {Abel }}(r)$ at $\beta=6.4,6.0$ and 5.8. At each $\beta$, all the data can be well fit with the pure Coulomb form with $\sigma=0$. (c) The demonstration of $V(r) \simeq V_{\text {Abel }}(r)+V_{\text {off }}(r)$ at $\beta=6.0$ (upper) and 6.4 (lower). All the figures are taken from Ref. [10].

We also examine the physical lattice-volume dependence of $\sigma_{\mathrm{Abel}} / \sigma$ in Fig. 2. Perfect Abelian dominance $\left(\sigma_{\mathrm{Abel}} / \sigma \simeq 1\right)$ seems to be realized when the spatial size $L a$ is larger than about $2 \mathrm{fm}$. 


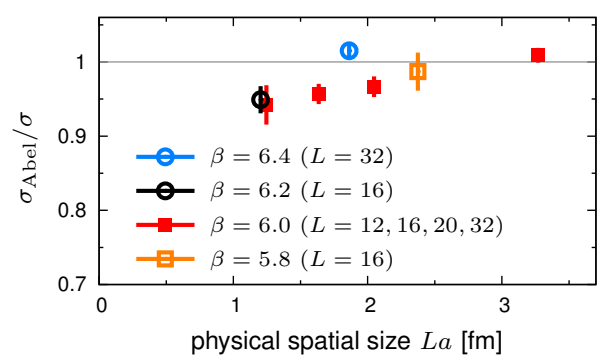

Figure 2: Physical spatial-size dependence of the ratio $\sigma_{\mathrm{Abel}} / \sigma$ of the QQ string tension and the Abelian one, taken from Ref. [9. (For $\beta=5.8$ and 6.0, both statistics and number of data in Ref. [9] are larger than those in Ref. [10].) Perfect Abelian dominance $\left(\sigma_{\mathrm{Abel}} / \sigma \simeq 1\right)$ is found for larger lattices with $L a \geq 2 \mathrm{fm}$.

\section{The baryonic three-quark potential}

In this section, we perform the accurate calculation of the baryonic three-quark (3Q) potential $V_{3 \mathrm{Q}}$ in $\mathrm{SU}(3)$ quenched lattice QCD with the standard plaquette action on the two lattices [9]:

i) $\beta=5.8$ on $16^{3} \times 32$, [i.e., $a=0.148(2) \mathrm{fm}$, the spatial volume $(L a)^{3}=(2.37(3) \mathrm{fm})^{3}$ ],

ii) $\beta=6.0$ on $20^{3} \times 32$, [i.e., $a=0.1022(5) \mathrm{fm}$, the spatial volume $(L a)^{3}=(2.05(1) \mathrm{fm})^{3}$ ].

The lattice spacing $a$ is determined from the string tension $\sigma=0.89 \mathrm{GeV} / \mathrm{fm}$ in the QQ potential. For $\beta=5.8$ and 6.0, we use 2000 and 1000 gauge configurations, respectively, which are taken every 500 sweeps after a thermalization of 20000 sweeps. The jackknife method is used for the error estimate.

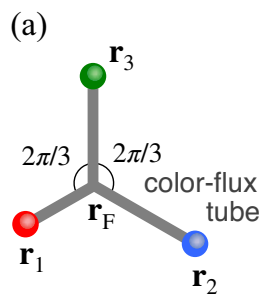

(b) $t$

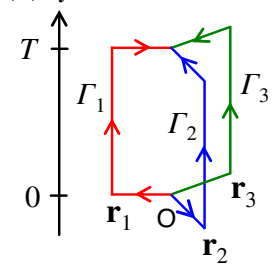

(c)

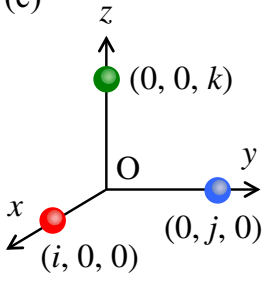

Figure 3: (a) The flux-tube configuration of the three-quark system with the minimal value of the total fluxtube length, $L_{\min } . \mathbf{r}_{F}$ is the Fermat point. (b) The trajectory of the 3Q Wilson loop $W_{3 \mathrm{Q}}$. (c) The location of the static three-quark sources in our lattice QCD calculation. These figures are taken from Ref. [9].

Similar to the case of the QQ potential $V(r)$, the color-singlet baryonic 3Q potential $V_{3 \mathrm{Q}}$ can be calculated from the 3Q Wilson loop $W_{3 \mathrm{Q}}$ as $[7,8]$

$$
V_{3 \mathrm{Q}}=-\lim _{T \rightarrow \infty} \frac{1}{T} \ln \left\langle W_{3 \mathrm{Q}}\left[U_{\mu}\right]\right\rangle, \quad W_{3 \mathrm{Q}}\left[U_{\mu}\right] \equiv \frac{1}{3 !} \sum_{a, b, c a^{\prime} b^{\prime} c^{\prime}} \varepsilon_{a b c} \varepsilon_{a^{\prime} b^{\prime} c^{\prime}} X_{1}^{a a^{\prime}} X_{2}^{b b^{\prime}} X_{3}^{c c^{\prime}} .
$$

Here, $X_{k} \equiv \prod_{\Gamma_{k}} U_{\mu}(s)$ is the path-ordered product of the link variables along the path $\Gamma_{k}$ in Fig. 3(b). We put three quarks on $(i, 0,0),(0, j, 0)$ and $(0,0, k)$ in $\mathbf{R}^{3}$ with $1 \leq i \leq j \leq k \leq L / 2$ in lattice units, as shown in Fig. 3(c), and deal with 101 and 211 different patterns of 3Q systems at $\beta=5.8$ and 6.0 , respectively, based on well-converged data of $\left\langle W_{3 \mathrm{Q}}\right\rangle$. For the accurate calculation of the 3Q potential with finite $T$, we apply the gauge-invariant smearing method [7 8], which enhances the ground-state component in the $3 \mathrm{Q}$ state in $\left\langle W_{3 \mathrm{Q}}\right\rangle$. 
Table 1: Fit analysis of inter-quark potentials in lattice units at $\beta=5.8$ on $16^{3} \times 32$ and $\beta=6.0$ on $20^{3} \times 32$.

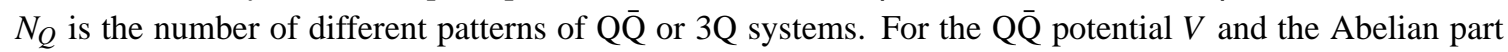
$V^{\text {Abel }}$, we list the best-fit parameter set $(\sigma, A)$ of the Coulomb-plus-linear ansatz. For the 3Q potential $V_{3 Q}$ and the Abelian part $V_{3 \mathrm{Q}}^{\text {Abel }}$, we list the best-fit parameter set $\left(\sigma_{3 \mathrm{Q}}, A_{3 \mathrm{Q}}\right)$ of the Y-ansatz. "3Q(equi.)" means the fit only for equilateral-triangle 3Q systems. The string tension ratio $\sigma^{\text {Abel }} / \sigma$ is also listed. (See Ref.[9].)

\begin{tabular}{cccccccc}
\hline$\beta$ & & $N_{\mathrm{Q}}$ & $\sigma$ & $A$ & $\sigma^{\text {Abel }}$ & $A^{\text {Abel }}$ & $\sigma^{\text {Abel }} / \sigma$ \\
\hline 5.8 & QQbar & 26 & $0.099(2)$ & $0.30(3)$ & $0.098(1)$ & $0.043(12)$ & $0.99(3)$ \\
& 3Q (equi.) & 5 & $0.097(1)$ & $0.118(3)$ & $0.098(3)$ & $-0.001(8)$ & $1.01(3)$ \\
& 3Q & 101 & $0.0997(4)$ & $0.109(1)$ & $0.0967(5)$ & $0.006(2)$ & $0.97(1)$ \\
\hline 6.0 & QQbar & 39 & $0.0472(6)$ & $0.289(10)$ & $0.0457(2)$ & $0.050(3)$ & $0.97(1)$ \\
& 3Q (equi.) & 8 & $0.0471(10)$ & $0.121(3)$ & $0.0455(12)$ & $0.014(4)$ & $0.97(3)$ \\
& 3Q & 211 & $0.0480(3)$ & $0.113(1)$ & $0.0456(2)$ & $0.013(1)$ & $0.95(1)$ \\
\hline
\end{tabular}

As the result, we find that the $3 \mathrm{Q}$ potential $V_{3 \mathrm{Q}}$ is fairly well reproduced by the Y-ansatz [7] 8 , i.e., one-gluon-exchange Coulomb plus Y-type linear potential,

$$
V_{3 \mathrm{Q}}\left(\mathbf{r}_{1}, \mathbf{r}_{2}, \mathbf{r}_{3}\right)=-\sum_{i<j} \frac{A_{3 \mathrm{Q}}}{\left|\mathbf{r}_{i}-\mathbf{r}_{j}\right|}+\sigma_{3 \mathrm{Q}} L_{\mathrm{min}}+C_{3 \mathrm{Q}}=-\frac{A_{3 \mathrm{Q}}}{R}+\sigma_{3 \mathrm{Q}} L_{\mathrm{min}}+C_{3 \mathrm{Q}}
$$

for all the distances of the $3 \mathrm{Q}$ systems $17,0,9$. Here, $\mathbf{r}_{1}, \mathbf{r}_{2}$ and $\mathbf{r}_{3}$ denote the three-quark positions, and $L_{\min }$ is the minimum flux-tube length connecting the three quarks, as shown in Fig. 3(a). Here, we have introduced a convenient variable $1 / R \equiv \sum_{i<j} 1 /\left|\mathbf{r}_{i}-\mathbf{r}_{j}\right|$.

Table 1 is a summary of the fit analysis for the $3 \mathrm{Q}$ potential $V_{3 \mathrm{Q}}$ with the Y-ansatz and the QQ potential $V$ with Eq. 3.2) in SU(3) lattice QCD at $\beta=5.8$ on $16^{3} \times 32$ and $\beta=6.0$ on $20^{3} \times 32$ [9].

As shown in Fig.3(a), the functional form (4.2) indicates the Y-shaped flux-tube formation in baryons. Actually, the Y-shaped flux-tube formation has been observed in the lattice QCD calculations on the action density in the presence of static three quarks [20, 21].

\section{Perfect Abelian dominance of quark confinement in baryons}

In this section, we investigate Abelian dominance of quark confinement in the 3Q system. Similarly to the QQ $\bar{Q}$ case, the MA-projected $3 \mathrm{Q}$ potential $V_{3 \mathrm{Q}}^{\text {Abel }}$ (Abelian part) can be calculated from the Abelian 3Q Wilson loop $W_{3 Q}\left[u_{\mu}\right]$ in the MA gauge:

$$
V_{3 \mathrm{Q}}^{\mathrm{Abel}}=-\lim _{T \rightarrow \infty} \frac{1}{T} \ln \left\langle W_{3 \mathrm{Q}}\left[u_{\mu}\right]\right\rangle,
$$

which is invariant under the residual Abelian gauge transformation.

Figure 4 shows the $3 \mathrm{Q}$ potential $V_{3 \mathrm{Q}}$ and its Abelian part $V_{3 \mathrm{Q}}^{\text {Abel }}$ plotted against $L_{\min }$ in $\mathrm{SU}(3)$ lattice QCD at $\beta=5.8$ on $16^{3} \times 32$ [9]. For comparison, we show in Fig.4(a) the QQ potential $V(r)$ and its Abelian part $V^{\mathrm{Abel}}(r)$, indicating perfect Abelian dominance of the string tension in mesons.

We note that the Abelian dominance of the QQ $\bar{Q}$ confinement force does not necessarily mean that of the 3Q confinement force, because one cannot superpose solutions in QCD even at the classical level. Indeed, any $3 \mathrm{Q}$ system cannot be described by the superposition of the interaction between two quarks, as is suggested from the functional form (4.2) of the $3 \mathrm{Q}$ potential [7, 8 ]. 

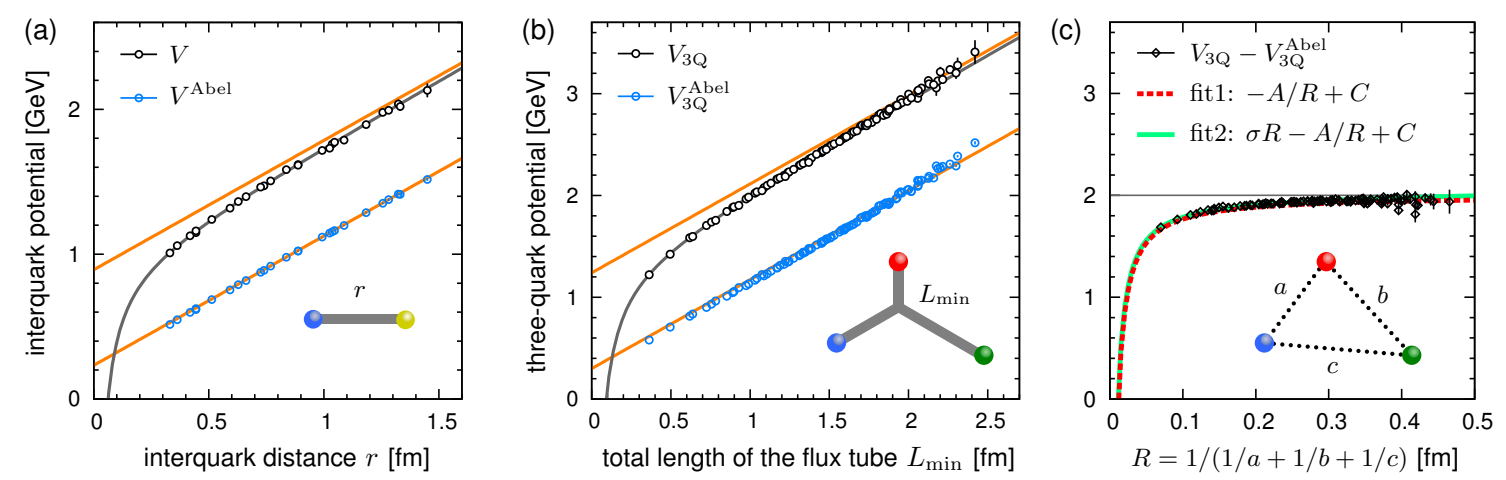

Figure 4: (a) The QQ potential $V(r)$ and its Abelian part $V^{\mathrm{Abel}}(r)$. (b) The 3Q potential $V_{3 \mathrm{Q}}$ (black) and its Abelian part $V_{3 \mathrm{Q}}^{\text {Abel }}$ (blue) in SU(3) lattice QCD at $\beta=5.8$ on $16^{3} \times 32$. For a rough indication, we add the best-fit Y-ansatz curve of the equilateral $3 \mathrm{Q}$ case for $V_{3 \mathrm{Q}}$ and $V_{3 \mathrm{Q}}^{\mathrm{Abel}}$, respectively. $\sigma_{3 \mathrm{Q}}$ and $\sigma_{3 \mathrm{Q}}^{\text {Abel }}$ correspond to the slopes of the parallel lines. (c) Fit analysis of $V_{3 Q}-V_{3 Q}^{\text {Abel }}$. The dashed curve is the pure Coulomb ansatz (5.3) with no string tension. (b) and (c) indicate $\sigma_{3 \mathrm{Q}} \simeq \sigma_{3 \mathrm{Q}}^{\mathrm{Abel}}$. These figures are taken from Ref. 9 .

We find that the Abelian part $V_{3 \mathrm{Q}}^{\text {Abel }}$ of the $3 \mathrm{Q}$ potential also takes the Y-ansatz [9],

$$
V_{3 \mathrm{Q}}^{\mathrm{Abel}}=-\frac{A_{3 \mathrm{Q}}^{\mathrm{Abel}}}{R}+\sigma_{3 \mathrm{Q}}^{\mathrm{Abel}} L_{\mathrm{min}}+C_{3 \mathrm{Q}}^{\mathrm{Abel}}
$$

with $1 / R \equiv \sum_{i<j} 1 /\left|\mathbf{r}_{i}-\mathbf{r}_{j}\right|$. Figure $4(\mathrm{~b})$ shows the $3 \mathrm{Q}$ potential $V_{3 \mathrm{Q}}$ and its Abelian part $V_{3 \mathrm{Q}}^{\text {Abel }}$ plotted against the total flux-tube length, $L_{\min }$. When the size of the 3Q system, $L_{\min } / 3$, is larger than $0.3 \mathrm{fm}, V_{3 \mathrm{Q}}$ is given by a Y-type linear potential, $\sigma_{3 \mathrm{Q}} L_{\mathrm{min}}+C_{3 \mathrm{Q}}$ (upper straight line in Fig.4(b)). Remarkably, the Abelian part $V^{\mathrm{Abel}}(r)$ obeys $\sigma_{3 \mathrm{Q}} L_{\mathrm{min}}+C_{3 \mathrm{Q}}^{\text {Abel }}$ (lower straight line in Fig.4(b)) at large distances, which means $\sigma_{3 \mathrm{Q}}^{\text {Abel }} \simeq \sigma_{3 \mathrm{Q}}$.

To demonstrate $\sigma_{3 \mathrm{Q}}^{\text {Abel }} \simeq \sigma_{3 \mathrm{Q}}$ conclusively, we investigate the difference $\Delta V_{3 \mathrm{Q}}$ between $V_{3 \mathrm{Q}}$ and $V_{3 \mathrm{Q}}^{\text {Abel }}$, as shown in Fig.4(c) [9]. If the Abelian dominance of the 3Q potential is exact, i.e., $\sigma_{3 \mathrm{Q}}^{\mathrm{Abel}}=\sigma_{3 \mathrm{Q}}, \Delta V_{3 \mathrm{Q}}$ is well reproduced by the pure Coulomb ansatz,

$$
\Delta V_{3 \mathrm{Q}} \equiv V_{3 \mathrm{Q}}-V_{3 \mathrm{Q}}^{\mathrm{Abel}}=-\frac{\Delta A_{3 \mathrm{Q}}}{R}+\Delta C_{3 \mathrm{Q}}
$$

where $\Delta A_{3 \mathrm{Q}} \equiv A_{3 \mathrm{Q}}-A_{3 \mathrm{Q}}^{\mathrm{Abel}}$ and $\Delta C_{3 \mathrm{Q}} \equiv C_{3 \mathrm{Q}}-C_{3 \mathrm{Q}}^{\mathrm{Abel}}$. In Fig.4(c), $\Delta V_{3 \mathrm{Q}}$ obeys a pure Coulomb form with no string tension, which is a clear evidence on the equivalence of $\sigma_{3 \mathrm{Q}}^{\text {Abel }}=\sigma_{3 \mathrm{Q}}$, with accuracy within a few percent deviation, i.e., perfect Abelian dominance of quark confinement in baryons.

In Table 1 , we summarize all the fit results for $V(r), V^{\text {Abel }}(r), V_{3 \mathrm{Q}}$ and $V_{3 \mathrm{Q}}^{\mathrm{Abel}}$ on both lattices at $\beta=5.8$ on $16^{3} \times 32$ and $\beta=6.0$ on $20^{3} \times 32$ [9]. Thus, we find perfect Abelian dominance for the string tension of $\mathrm{QQ}$ and $3 \mathrm{Q}$ potentials: $\sigma_{\mathrm{QQ}} \simeq \sigma_{\mathrm{Q} \overline{\mathrm{Q}}}^{\mathrm{Abel}} \simeq \sigma_{3 \mathrm{Q}} \simeq \sigma_{3 \mathrm{Q}}^{\mathrm{Abel}}$.

\section{Summary and concluding remarks}

We have studied the baryonic 3Q potential in SU(3) quenched lattice QCD with $\beta=5.8$ on $16^{3} \times 32$ and $\beta=6.0$ on $20^{3} \times 32$ for more than 300 different patterns of $3 Q$ systems in total, using 1000-2000 gauge configurations. For all the distances, we have found that the $3 \mathrm{Q}$ potential is fairly well described by the Y-ansatz, i.e., one-gluon-exchange Coulomb plus Y-type linear potential [9]. 
We have also investigated MA projection of quark confinement in both mesons and baryons, and have found perfect Abelian dominance of the string tension, $\sigma_{\mathrm{QQ}} \simeq \sigma_{\mathrm{QQ}}^{\mathrm{Abel}} \simeq \sigma_{3 \mathrm{Q}} \simeq \sigma_{3 \mathrm{Q}}^{\text {Abel }}$, in $\mathrm{QQ}$ and 3Q potentials [9]. Thus, in spite of the non-Abelian nature of QCD, quark confinement in hadrons is entirely and universally kept in the Abelian sector of QCD in the MA gauge.

\section{Acknowledgments}

H. S. sincerely thanks Yoichiro Nambu for his interest to our studies and valuable suggestions in old days. H. S. also thanks V. G. Bornyakov for his useful advices. This work is supported in part by the Grants-in-Aid for Scientific Research [15K05076, 15K17725] from Japan Society for the Promotion of Science. The lattice calculations were done on NEC-SX8R at Osaka University.

\section{References}

[1] Y. Nambu, in Preludes in Theoretical Physics, in honor of V. F. Weisskopf (North-Holland, 1966).

[2] M. Y. Han and Y. Nambu, Phys. Rev. 139, B1006 (1965).

[3] D. J. Gross and F. Wilczek, Phys. Rev. Lett. 30, 1343 (1973); H. D. Politzer, PRL 30, 1346 (1973).

[4] Y. Nambu and G. Jona-Lasinio, Phys. Rev. 122, 345 (1961); Phys. Rev. 124, 246 (1961).

[5] Y. Nambu, Phys. Rev. D10, 4262 (1974); G. 't Hooft, in High Energy Physics, (Editorice Compositori, Bologna, 1975); S. Mandelstam, Phys. Rept. 23, 245 (1976).

[6] H. J. Rothe, Lattice Gauge Theories, 4th ed. (World Scientific, 2012), and its references.

[7] T. T. Takahashi, H. Matsufuru, Y. Nemoto, and H. Suganuma, Phys. Rev. Lett. 86, 18 (2001); T. T. Takahashi, H. Suganuma, Y. Nemoto, and H. Matsufuru, Phys. Rev. D65, 114509 (2002).

[8] T. T. Takahashi and H. Suganuma, Phys. Rev. Lett. 90, 182001 (2003); Phys. Rev. D70, 074506 (2004); F. Okiharu, H. Suganuma, and T. T. Takahashi, Phys. Rev. D72, 014505 (2005).

[9] N. Sakumichi and H. Suganuma, Phys. Rev. D92, 034511 (2015).

[10] N. Sakumichi and H. Suganuma, Phys. Rev. D90, 111501(R) (2014).

[11] Y. Nambu, in Symmetries and Quark Models (Wayne State Univ., 1969); Lecture Notes at the Copenhagen Symposium (1970).

[12] H. Ichie and H. Suganuma, Nucl. Phys. B548, 365 (1999); Nucl. Phys. B574, 70 (2000).

[13] G. 't Hooft, Nucl. Phys. B190, 455 (1981).

[14] Z. F. Ezawa and A. Iwazaki, Phys. Rev. D25, 2681 (1982).

[15] A. S. Kronfeld, G. Schierholz, and U.-J. Wiese, Nucl. Phys. B293 461 (1987); A. S. Kronfeld, M. L. Laursen, G. Schierholz, and U.-J. Wiese, Phys. Lett. B198, 516 (1987).

[16] T. Suzuki and I. Yotsuyanagi, Phys. Rev. D42, 4257(R) (1990).

[17] K. Amemiya and H. Suganuma, Phys. Rev. D60, 114509 (1999); S. Gongyo and H. Suganuma, Phys. Rev. D87, 074506 (2013); S. Gongyo, T. Iritani, and H. Suganuma, Phys. Rev. D86, 094018 (2012).

[18] J. D. Stack, S. D. Neiman, and R. J. Wensley, Phys. Rev. D50, 3399 (1994).

[19] J. D. Stack, W. W. Tucker, and R. J. Wensley, Nucl. Phys. B639, 203 (2002).

[20] V. G. Bornyakov et al. (DIK Coll.), Phys. Rev. D70, 054506 (2004); Phys. Rev D70, 074511 (2004).

[21] H. Ichie, V. Bornyakov, T. Streuer, and G. Schierholz, Nucl. Phys. A721, 899 (2003). 